\begin{tabular}{lll} 
Abstract P021 Table & \multicolumn{2}{l}{ Results: referral numbers } \\
\hline & 2019 & 2020 \\
\hline Jan-March & 17 & 16 \\
April-June & 22 & 11 \\
July-Sept & 17 & 25 \\
Oct-Dec & 26 & 28 \\
\hline
\end{tabular}

36 patients $(45 \%)$ were offered surgical (6) or radiological (30) therapy in 2020 compared to 32 in 2019.

Similar radiological therapy was recommended both years. The main difference in surgical treatment was that only 3 resections were undertaken in 2020, compared to 7 in 2019. In 2020, 2 patients were referred for transplant assessment in November and December 2020 (none referred earlier in year), with 3 referred in 2019.

Palliative care was recommended for 44 (55\%) patients in 2020 , which was similar to $48(58 \%)$ of patients in 2019 . In 2020, 10 patients were referred for systemic therapy (17 in 2019). 2 patients received sorafenib compared to 7 the previous year.

Of those referred for palliative care, 17 had treatable disease but the patient was not fit for treatment while another 17 had extensive/metastatic disease and so were recommended for supportive care.

Conclusion COVID 19 may have had an impact on referral patterns and HCC screening programs in 2020. However, the total number of HCC cases referred to the regional HPB MDM in 2020 was as expected and the treatment options recommended were very similar to 2019. The only substantial difference was a reduction in the number of patients who were commenced on systemic therapy.

\section{P022 CLINICAL PRESENTATION AND OUTCOMES OF WILSON'S DISEASE: A SINGLE CENTRE COHORT STUDY}

Akhilesh Mulay*, James Dooley, Emmanouil Tsochatzis. UCL Institute for Liver and Digestive Health, Royal Free Hospital and UCL, London, UK

\subsection{6/gutjnl-2021-BASL.31}

Introduction Wilson's Disease is a disorder of copper homeostasis leading to hepatic and neuropsychiatric disease. Clinical data on these patients are limited due to low disease frequency. This report explores the presentation, management and outcomes of a large cohort of patients with Wilson's disease including pregnancy outcomes.

Methods A retrospective analysis was performed on 63 patients with Wilson's disease managed at the Royal Free Hospital, London, between 2012 and 2021. Clinical data were collected on presenting features, investigations, treatments and outcomes.

Results The mean age at diagnosis was 20 years (range 7-54 years) with 38 patients presenting due to liver involvement, 13 due to neuropsychiatric disease, 4 with both and 8 due to family screening. Of those presenting with liver involvement, $43 \%$ had asymptomatic liver disease with incidentally identified abnormal liver blood tests. The mean delay between onset of symptoms and diagnosis was 2 years (max. 8 years). During disease management, 31 patients received one type of drug treatment while 32 received more than one. In total, 48 patients received Penicillamine; of these, 11 were noncompliant to treatment, 16 experienced adverse effects and 21 stopped treatment. The most common adverse effect was skin changes (38\% of patients). In patients who stopped Penicillamine, $67 \%$ did so due to adverse effects. Trientine was given to 25 patients ( 1 noncompliant, 2 with adverse effects and 6 stopped treatment) while Zinc was given to 22 patients (4 noncompliant, 3 with adverse effects and 8 stopped treatment). At latest follow-up (mean 10.2 years), 92\% of patients with symptomatic liver disease, $87 \%$ with neurological disease and $89 \%$ with psychiatric symptoms had clinically improved or were stable. In patients with no psychiatric involvement at diagnosis, 26\% developed new psychiatric symptoms. Four patients underwent liver transplant, 3 due to decompensated cirrhosis and 1 due to acute liver failure. There were 3 deaths, of which 2 were due to liver-related complications. Seven patients had 12 pregnancies; 10 were on Penicillamine and 2 were on Zinc therapy throughout the pregnancy. Of those pregnancies on Penicillamine, 8 were normal, 1 was a miscarriage and 1 was a termination. Both pregnancies on Zinc were normal.

Conclusions The majority of patients with Wilson's disease who present primarily with liver involvement are asymptomatic with incidentally identified abnormal liver blood tests. New psychiatric symptoms commonly develop after diagnosis and may warrant more proactive psychological input. Penicillamine and Zinc appear to be safe in pregnancy.

\section{P023 THE EFFECTIVE USE OF BEZAFIBRATE AS A SECOND LINE THERAPY FOR PRIMARY BILIARY CHOLANGITIS}

Adam Howden*, Talal Valliani. North Bristol NHS Trust Liver Unit, UK

\subsection{6/gutjnl-2021-BASL.32}

Background Bezafibrate, a peroxisome proliferator-activated receptor alpha agonist, is not often used in the UK for treatment of PBC (primary biliary cholangitis) in patients who don't respond to ursodeoxycholic acid (UDCA) because it is an unlicensed therapy for this condition. In our liver unit we have used this treatment since 2014 .

Methods This retrospective case series included all patients who were commenced on bezafibrate from January 2014 to March 2020. 25 patients were listed from our pharmacy database and notes reviewed using a data collection tool. Biochemical and clinical outcomes were recorded over a 12 month period following initiation of bezafibrate in combination with UDCA.

10 patients were excluded due to either: non-compliance; a revised diagnosis of an overlap syndrome or primary sclerosing cholangitis; less than 12 months duration of bezafibrate therapy; lost to follow-up; not fulfilling PBC diagnostic criteria as per The British Society Gastroenterology/UK-PBC management guidelines. ${ }^{1}$

An inadequate response to UDCA therapy was defined by the 'Toronto' biochemical stratification as a serum alkaline phosphatase (ALP) level above $1.67 \mathrm{x}$ upper limit of normal (normal range $30-130 \mathrm{U} / \mathrm{L}$ ). This value is $217 \mathrm{U} / \mathrm{L}$ in our unit.

Results The 15 patients were all female with a median age at diagnosis of $49.67 \%$ were positive for anti-mitochondrial antibody or PBC specific anti-nuclear antibody. 33\% had consistent liver histology and the median ALP was $477 \mathrm{U} / \mathrm{L}$ at 


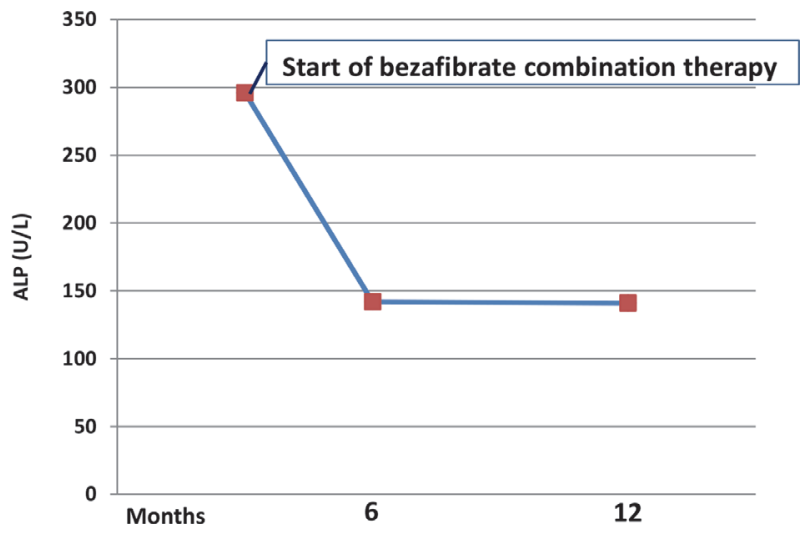

Abstract P023 Figure 1

diagnosis. The mean duration of UDCA monotherapy was 62 months with a median dose of $19 \mathrm{mg} / \mathrm{kg} /$ day (range 11-26). At the time of commencing bezafibrate, the median ALP was $296 \mathrm{U} / \mathrm{L}$ (mean $301 \mathrm{U} / \mathrm{L}$ ), mean ALT was $52 \mathrm{U} / \mathrm{L}$ and mean albumin was $36 \mathrm{~g} / \mathrm{L}$. The median bilirubin was $8 \mathrm{umol} / \mathrm{L}$ (range 5-37) and no patients had evidence of clinically significant portal hypertension. 3 patients had radiological evidence of cirrhosis.

The bezafibrate was continued at $400 \mathrm{mg} /$ day in 14 patients and $200 \mathrm{mg} /$ day in 1 patient who developed myalgia. Figure 1 shows the median ALP at the start of bezafibrate therapy and an improvement in median ALP at 6 and 12 months of therapy. After 12 months, the median ALP was $141 \mathrm{U} / \mathrm{L}$ with a mean of $178 \mathrm{U} / \mathrm{L} .80 \%$ of patients $(12 / 15)$ achieved an ALP of $<217 \mathrm{U} / \mathrm{L}$. Of the 3 who didn't; 1 died from metastatic breast cancer; 1 was referred for liver transplantation and 1 has commenced obeticholic acid. No patients developed renal impairment.

Conclusion Our case series demonstrates that bezafibrate is a highly effective second line therapy for $\mathrm{PBC}$, in combination with UDCA, for patients who don't have an adequate biochemical response with monotherapy.

\section{REFERENCE}

1. Hirschfield GM, Dyson JK, Alexander GJM, et al. The british society of gastroenterology/UK-PBC primary biliary cholangitis treatment and management guidelines. Gut 2018;67:1568-1594.

\section{P024 KEYNOTE-937 TRIAL IN PROGRESS: ADJUVANT PEMBROLIZUMAB FOR HEPATOCELLULAR CARCINOMA AND COMPLETE RADIOLOGIC RESPONSE AFTER SURGICAL RESECTION OR LOCAL ABLATION}

${ }^{1}$ Lipika Goyal ${ }^{*},{ }^{2}$ Arndt Vogel, ${ }^{1}$ Andrew X Zhu, ${ }^{3}$ Ann-Lii Cheng, ${ }^{4}$ Thomas Yau, ${ }^{5}$ Jian Zhou, ${ }^{1}$ Raul N Uppot, ${ }^{6}$ Eunhee Kim, ${ }^{6}$ Usha Malhotra, ${ }^{6}$ Abby B Siegel, ${ }^{7}$ Masatoshi Kudo. ${ }^{1}$ Massachusetts General Hospital Cancer Center, Harvard Medical School, Boston, USA; ${ }^{2}$ Hannover Medical School, Hannover, Germany; ${ }^{3}$ National Taiwan University Cancer Center and National Taiwan University Hospital, Taipei, Taiwan; ${ }^{4}$ University of Hong Kong, Queen Mary Hospital, Hong Kong, China; ${ }^{5}$ Zhongshan Hospital, Fudan University, Shanghai, China; ${ }^{6}$ Merck and Co., Inc., Kenilworth, USA; ${ }^{7}$ School of Medicine, Kindai University, Osaka, Japan

\subsection{6/gutjnl-2021-BASL.33}

Introduction Hepatocellular carcinoma (HCC) is a leading cause of cancer-related deaths worldwide. Surgical resection and local ablation are potentially curative options for patients with HCC; however, tumor recurrence is not uncommon. The lack of a standard-of-care adjuvant therapy for HCC highlights an urgent therapeutic need to prevent disease recurrence and improve overall survival. Pembrolizumab, a PD-1 inhibitor, has shown evidence of a favorable benefit-to-risk profile in the adjuvant setting for many tumor types but has not been investigated in HCC. The KEYNOTE-937 study (NCT03867084) will evaluate the safety and efficacy of pembrolizumab versus placebo as adjuvant therapy in patients with HCC who had a complete radiologic response after surgical resection or local ablation.

Methods KEYNOTE-937 is a randomized, double-blind, phase 3 study. Adults with confirmed HCC, complete radiologic response after surgical resection or local ablation, Eastern Cooperative Oncology Group performance status of 0 or 1 , and Child-Pugh liver class A are eligible. Patients with past or ongoing hepatitis $\mathrm{C}$ or controlled hepatitis $\mathrm{B}$ virus infection may be enrolled if they meet prespecified criteria. Patients will be randomly assigned $1: 1$ to receive pembrolizumab $200 \mathrm{mg}$ intravenously or placebo intravenously every 3 weeks for up to 17 cycles or until disease recurrence, unacceptable toxicity, or withdrawal and will be stratified by geographic region, prior local therapy (resection vs ablation), recurrence risk, and alpha-fetoprotein level at diagnosis. Coprimary end points are recurrence-free survival and overall survival; secondary end points are safety and tolerability (graded per National Cancer Institute Common Terminology Criteria for Adverse Events, version 4.0) and health-related quality of life; exploratory end points include distant metastasis-free survival, time to recurrence, and genomic, metabolic, and/or proteomic biomarkers. Tumor imaging will be assessed until recurrence, and adverse events will be recorded up to 30 days after the last dose (90 days for serious adverse events).

Results Recruitment began in May 2019. The planned sample size is 950 patients.

Discussion Results of the KEYNOTE-937 study will show the efficacy and safety of pembrolizumab as adjuvant therapy for patients with HCC who had a complete radiologic response after surgical resection or local ablation.

\section{P025 OSHI (OPEN SOURCE HEALING INITIATIVE): AN EXAMPLE OF PATIENT-LED INNOVATION IN LIVER SERVICES}

James Halls*, Dianne Backhouse, Neil Gordon, Lynsey Corless. Hull University Teaching Hospitals, UK

\subsection{6/gutjnl-2021-BASL.34}

Introduction Alcohol use disorders and alcohol related liver disease have a significant adverse impact on individuals, healthcare and society. Although abstinence is highly effective, engagement with traditional community alcohol support services (CASS) is poor, thereby reducing opportunities to improve outcomes. Reasons for this are complex, but inflexibility of access to existing services is important, and many patients do not feel comfortable discussing alcohol in clinical/group settings.

To address these potential barriers to engagement, we introduced a personalized intervention. OSHI, which stands for Open Source Healing Initiative as well as being the Japanese word for 'friend that supports', was developed and delivered by a patient who joined our team as a peer-support volunteer. 